\title{
Immunization with a recombinant subunit OspA vaccine markedly impacts the rate of newly acquired Borrelia burgdorferi infections in client-owned dogs living in a coastal community in Maine, USA
}

\author{
Andrew K Eschner ${ }^{1 *}$ and Kristen Mugnai ${ }^{2}$
}

\begin{abstract}
Background: In North America, Borrelia burgdorferi is the causative bacterial agent of canine Lyme borreliosis and is transmitted following prolonged attachment and feeding of vector ticks, Ixodes scapularis or Ixodes pacificus. Its prevention is predicated upon tick-avoidance, effective on-animal tick control and effective immunization strategies. The purpose of this study is to characterize dogs that are newly seropositive for Borrelia burgdorferi infection in relation to compliant use of a recombinant OspA canine Lyme borreliosis vaccine. Specifically, Preventive Fractions (PF) and Risk Ratios (RR) associated with the degree of vaccine compliancy (complete versus incomplete) are determined.
\end{abstract}

Methods: 6,202 dogs were tested over a five year period in a single veterinary hospital utilizing a non-adjuvanted, recombinant OspA vaccine according to a 0, 1, 6 month (then yearly) protocol. Rates of newly acquired "Lyme-positive" antibody test results were compared between protocol compliant and poorly compliant (incompletely and/or non-vaccinated) dogs.

Results: Over the five-year span, one percent (range 0.39 - 1.3) of protocol compliant vaccinated, previously antibody negative dogs became seropositive for infection. Approximately twenty-one percent (range 16.8 - 33.3) of incompletely vaccinated dogs became positive for infection-specific antibodies. The Preventative Fraction for testing positive for antibodies specific for infection with Borrelia burgdorferi in any given year based on optimal vaccine compliance was, on average, 95.3\% (range 93.29 - 98.08). The Risk Ratio for becoming infected with Borrelia burgdorferi antibodies in any given year if vaccine non-compliant was 21.41 (range 14.9 - 52.1). There was a high statistically significant relationship $(p=<0.0001)$ in the observed data in terms of vaccination protocol compliance and the probability of Borrelia burgdorferi infection in each of the five years under study.

Conclusions: The recombinant outer surface protein A (rOspA) vaccine for dogs is highly effective in preventing new seropositive cases of Borrelia burgdoferi infection over a five-year period in dogs living in an endemic area. Dogs that were vaccine protocol-compliant were significantly less likely to become infected (as indirectly assessed by antibody) with the agent of canine Lyme borreliosis as measured by Preventive Fraction and Risk Ratio calculations.

Keywords: Borrelia burgdorferi, Canine, Lyme, Borreliosis, Vaccine, Recombinant, OspA, Veterinary, Antibodies

\footnotetext{
* Correspondence: drandrew.eschner@merial.com

'Merial Limited, 3239 Satellite Boulevard NW, Duluth, GA 30096, USA

Full list of author information is available at the end of the article
} 


\section{Background}

\section{Canine lyme borreliosis}

In the United States, canine Lyme borreliosis was first described in 1984 [1]. Infection and disease results from the successful transmission of the spirochete, Borrelia burgdorferi, from an infected tick to a dog [1]. The ecology, biology and disease manifestations of Borrelia burgdorferi, as well as its sister species in Europe (B. afzellii and B. garinii) and its tick vector in the Ixodes genus have been thoroughly described elsewhere in the literature [2-5].

Four brands, representing two distinct classes of vaccines, are available for use in dogs in the United States for the purpose of preventing Lyme borreliosis. To date, five placebo-controlled laboratory studies pertaining to commercialized whole-cell Lyme borreliosis bacterins and recombinant OspA vaccines for dogs have been published [6-10]. In addition, four published papers have explored the performance of canine Lyme disease vaccines in the private, clinical practice setting [11-14]. Testing parameters, including time frames, site locations, and sample size were dissimilar within all of these studies so direct comparison of vaccine efficacy are not feasible. While whole cell bacterins are appreciably efficacious depending on which parameter you are interested in, reports have described the occasional occurrence of post-vaccinal "Lyme-like" clinical signs in some recently vaccinated, uninfected dogs $[7,15,16]$. These signs have been putatively associated with the wider array of proinflammatory outer surface Borrelia proteins contained within bacterins $[15,16]$. In an effort to decrease the adverse reaction profile and remedy the occasional diagnostic conundrums posed by whole cell bacterins for Lyme borreliosis, a Type I recombinant OspA (rOspA) subunit extract was licensed and marketed for canine use in the United States in 1996. This vaccine was the first Lyme disease vaccine to be licensed by the USDA demonstrating a 1-year duration of immunity (DOI) after use of a natural tick challenge model [9]. Efficacy of the rOspA vaccine was based on the ability of the vaccine to prevent spirochete transmission as assessed by re-isolation of spirochetes from skin biopsies of vaccinated and non-vaccinated control dogs. In 2000, a randomized, placebo-controlled study explored the effectiveness of the recombinant OspA vaccine (Recombitek ${ }^{\ominus}$ Lyme, Merial) and demonstrated that non-adjuvanted recombinant OspA completely prevented B. burgdorferi infection in vaccinated dogs [10].

The following analysis offers a comprehensive look at the performance of the recombinant OspA Lyme-disease vaccine over five years of continuous use in a busy, 3doctor small (companion) animal practice in Wiscasset, Maine. Since 2009, the practice has been vaccinating dogs to prevent canine Lyme borreliosis utilizing the non-adjuvanted, recombinant OspA subunit vaccine
(Recombitek ${ }^{\circledR}$ Lyme, Merial) using an alternate dose veterinary protocol first suggested by Töpfer and Straubinger [17]. These authors observed that "regardless of vaccine used, the third vaccination induced significantly higher antibody levels." This protocol modification suggestion was predicated on earlier work in human medicine showing that the addition of a third dose of vaccine within 6 months of starting the basic immunization protocol could increase the proportion of protected individuals by a factor of almost 2-fold [18]. With emphasis on preventing canine Lyme borreliosis utilizing the Töpfer-Straubinger vaccine protocol recommendations, this Maine veterinary practice advanced the initial annual Lyme disease booster vaccine forward in time by six months. The goal was to raise antibody levels to their maximum more quickly within a dog's first year and thus they adopted the $0,1,6$ month vaccine protocol instead of the traditional 0, 1, 12 month framework. Accordingly, the veterinary hospital described in this paper chose to include the recombinant OspA vaccine as a $C O R E$ vaccine in their vaccine repertoire beginning at 9 weeks of age, utilize a modified protocol and to booster yearly in order to minimize the rate of new $B$. burgdorferi infections in their canine patients. Diagnostic assessment of Lyme 'positive' or 'negative' was made using a popular test kit $\left(\mathrm{SNAP}^{\oplus}\right)$ manufactured by IDEXX Laboratories. SNAP ${ }^{\circledR}$ is a proprietary rapid, patient-side, lateral flow ELISA technology platform that can detect antibody or antigen to a variety of vector-borne parasites including C6 antibody to Borrelia burgdorferi, antibody to Ehrlichia canis, and antigen of the canine heartworm Dirofilaria immitis (SNAP ${ }^{\circ}$ 3Dx). Currently, antibodies to Anaplasma phagocytophilum and Ehrlichia ewingii can also be detected (SNAP ${ }^{\oplus}$ 4Dx Plus). Further information pertaining to product validation, testing and sensitivity/specificity can be read elsewhere [19].

\section{Methods}

From 2009-2010, the hospital utilized SNAP 3Dx; from 2011-2012, SNAP 4Dx; and for 2013, SNAP 4Dx Plus; the change in kit-type usage over time was the result of increased pathogen testing capabilities by the kit itself and each successive new product was incorporated soon after market entry. The analysis of new Lyme infection rates in vaccinated versus incompletely vaccinated dogs was conducted annually at the conclusion of each year beginning in 2009 until the end of 2013. Utilizing the hospital's veterinary management software (AVImark ${ }^{\oplus}$, Piedmont, MO), the results of all SNAP tests conducted in dogs during the analysis year were tallied. Dogs that tested SNAP positive for infection-specific B. burgdorferi-antibody were characterized according to vaccine status and 'age' of their positive-status. For the analysis, 
the following parameters/definitions were set. TEST “AGE”: A 'NEW' SNAP B. burgdorferi-infection positive result ('blue-dot') was defined as a positive-test on a dog that had tested negative on its most previously recorded test. An ' $O L D$ ' SNAP $B b$ infection-positive was defined as a repeat positive-test in a dog that also tested positive on its most recent previous test. For purposes of this retrospective study, dogs labelled as 'OLD-positive' were not counted in the analysis of new infection rates in the universe of vaccinated or non-vaccinated dogs within that year. VACCINE STATUS: A 'FULLY' vaccinated dog was defined as a dog that had adhered to the hospital vaccine recommendation for interval and compliance for at least one year following a negative SNAP test for B. burgdorferi antibodies. An 'INCOMPLETELY vaccinated' dog was segmented into the degree of incompleteness. 'PARTIALLY vaccinated' was defined as having received an initial vaccine with no boosters or having completed its first series of two immunizations with no subsequent booster. 'NON-VACCINATED' was defined as having had no vaccines in its life or being greater than 15 months beyond its regularly scheduled yearly booster.

\section{Analysis of new-positive infection rates}

New SNAP B. burgdorferi-infection positive test results were sorted based on the occurrence in the 'Fully' vaccinated, protocol-compliant group and the 'incompletely' vaccinated/non-vaccinated dogs. For the purpose of this study, Infection rate (IR) differences were determined by examining the proportion of new-positive test results in fully vaccinated dogs amongst all fully vaccinated dogs tested that year. Likewise, the number of new-positive test results in incompletely vaccinated dogs (either partial or non-vaccinated) was compared to the total of all incompletely vaccinated dogs.

Risk Ratio(RR), the ratio of the probability of an event (for example, developing a positive test) occurring in an incompletely vaccinated group to the probability of the event occurring in a comparison group (Fully-vaccinated group), were calculated for each year 2009-2013 according to the following equation [20].

$$
\text { Risk Ratio }=\left(\mathbf{C I}_{\mathbf{u}}\right) /\left(\mathbf{C I}_{\mathbf{e}}\right)
$$

[where $\mathrm{CI}_{\mathrm{u}}$ is the cumulative incidence in the group 'unexposed' to Full vaccination and $\mathrm{CI}_{\mathrm{e}}$ is the cumulative incidence in the group 'exposed' to Full vaccination].

Preventive Fraction (PF) for Benefit of Vaccination, the percentage of infection reduction in the group exposed to Full vaccination that can actually be attributed to the 'benefit of full vaccine exposure, was calculated each year by dividing the risk reduction between two groups (incidence in unexposed (incompletely vaccinated) minus incidence in exposed (Fully vaccinated) group by the incidence in the unexposed (expressed as a percent) according to the following equation [20].

$$
\text { Preventive Fraction }=\mathbf{P F}=\left[\left(\mathbf{C I}_{\mathbf{u}}\right)-\left(\mathbf{C I}_{\mathbf{e}}\right)\right] /\left(\mathbf{C I}_{\mathbf{u}}\right) \mathbf{x} 100
$$

[where $\mathrm{CI}_{\mathrm{u}}$ is the cumulative incidence in the group 'unexposed' to Full vaccination and $\mathrm{CI}_{\mathrm{e}}$ is the cumulative incidence in the group 'exposed' to Full vaccination].

\section{Results}

During the five years of analysis (2009-2013), 6,202 SNAP $^{\circ}$ tests were completed on 4,551 well vaccinated and 1,651 incompletely vaccinated dogs that had previously been characterized as either SNAP 'positive' or 'negative' (prior to study onset). In total, 835 tests (13.5\%) were positive indicating the presence of C6 antibodies specific for infection with Borrelia burgdorferi. Inspection of patient records revealed that 432 of the positive tests were in dogs that had also tested positive on their most recent previous test, therefore labeled as 'OLD' positives, and were excluded from the exercise of determining the rate of new infections in vaccinated dogs. Four hundred three dogs were $N E W$ positives in the year tested (had tested negative on their most recent previous test), and made up $6.5 \%$ of all SNAP tests performed. In the population of 6,202 dogs tested during 2009-2013, 4551 were fully vaccinated against canine Lyme borreliosis and compliant with hospital vaccine-protocol recommendations [Table 1]. There were 46 new positive tests among these fully vaccinated dogs. One thousand six hundred fifty one tests occurred in incompletely vaccinated dogs and among these, 357 were newly positive.

The summary of the cumulative test results (20092013) is displayed [Table 2]. The conditional probabilities for each vaccination condition are displayed within parentheses under the counts. Given that a dog is fully vaccinated, the probability of this dog testing positive in our sample is $1.01 \%$. Similarly, given a dog is incompletely vaccinated, the probability of this dog testing positive in our sample is $21.62 \%$ [Table 2]. This appears to be a large practical difference in the percentage of dogs testing positive. However, because dogs are supposed to be tested annually for Lyme borreliosis, it is likely that the same dogs are being tested in different years. Therefore, in order to avoid artificially increasing the sample size and introducing dependence into the observations (since results from one dog may be related across different years), data were analyzed in yearly increments [21].

\section{Statistical analysis}

The summary of test results for each year beginning 2009 through 2013 is displayed [Table 3]. For 2009, the Chi-squared test statistic is 130.95 with a corresponding 
Table 1 Tabulation of yearly testing results for antibodies directed against $\mathrm{C} 6$ antigen of Borrelia burgdorferi in dogs utilizing the SNAP ${ }^{\circledR} 3 D x^{\circledR}$ or $4 D x^{\circledR}$ test kit

\begin{tabular}{|c|c|c|c|c|c|c|}
\hline & 2009 & 2010 & 2011 & 2012 & 2013 & Cumulative \\
\hline SNAP tests utilized & 1391 & 1346 & 1266 & 1127 & 1072 & 6202 \\
\hline SNAP B. burgdorferi-infection positive results & 170 & 208 & 156 & 154 & 147 & 835 \\
\hline Old POS (+) & 88 & 103 & 85 & 75 & 81 & 432 \\
\hline New POS (+) & 82 & 105 & 71 & 79 & 66 & 403 \\
\hline \multicolumn{7}{|l|}{ Vaccine compliance status of tested dogs } \\
\hline Fully vaccinated dogs tested & 970 & 1053 & 933 & 833 & 762 & 4551 \\
\hline New POS $(+)$ & 11 & 11 & 10 & 11 & 3 & 46 \\
\hline Negative $(-)$ & 959 & 1042 & 923 & 822 & 759 & 4505 \\
\hline Incompletely vaccinated dogs tested (also includes non-vaccinated) & 421 & 293 & 333 & 294 & 310 & 1651 \\
\hline Total new $(+)$ : (includes dogs with unknown vaccine status)* & 71 & 94 & 61 & 68 & 63 & 357 \\
\hline New POS (+) among NON-vaccinated & 56 & 76 & 55 & 55 & 49 & 291 \\
\hline New POS (+) among partially vaccinated & 15 & 18 & 6 & 13 & 14 & 66 \\
\hline Negative $(-)$ & 350 & 199 & 272 & 226 & 247 & 1294 \\
\hline
\end{tabular}

*Dogs with unknown vaccine status grouped (conservatively) with 'Incompletely Vaccinated Group': 2009 (12); 2010 (11), 2011 (0), 2012 (0), 2013 (1).

$p$-value less than 0.0001 . Briefly this means that if there is truly no relationship between the SNAP test results for fully and incompletely vaccinated groups, the probability of collecting a sample with a relationship as strong as the one in our sample is less than 0.0001 [21]. For $2010-$ 2013, the Chi-squared test statistics were 307.01, 137.89, 158.56 , and 151.47 , respectively with a corresponding $p$ values less than 0.0001 for each statistic [21].

The Risk Ratio (RR) describing the probability of Borrelia burgdorferi infection (as determined by SNAP) in incompletely vaccinated dogs compared to 'fully' vaccinated dogs between 2009 and 2013 averaged 21.4 (range 14.8-52.1) [Table 3]. The Preventative Fractions (PF) for prevention of a positive test result for each year beginning in 2009 through 2013 were 93.29, 96.76, 94.16, 94.29, 98.08, respectively [Table 3].

\section{Discussion}

Four different canine Lyme borreliosis vaccines represented by six distinct brand names have been licensed by

Table 2 Cumulative summary of SNAP test results for Borrelia burgdorferi-specific antibodies in completely and incompletely vaccinated dogs (2009-2013)

\begin{tabular}{llll}
\hline & Positive & Negative & Total \\
\hline Completely vaccinated $^{*}$ & 46 & 4505 & 4551 \\
& $(1.01 \%)$ & $(98.99 \%)$ & \\
Incompletely vaccinated $^{*}$ & 357 & 1294 & 1651 \\
& $(21.62 \%)$ & $(78.38 \%)$ & \\
Total & 403 & 5799 & 6202 \\
\hline
\end{tabular}

*See working definition of completely and incompletely vaccinated dogs in Methods section. the USDA from 1992-2009. Immunizing against canine Lyme borreliosis can be achieved by delivering either a whole-cell bacterial lysate (bacterin) or a recombinant subunit vaccine containing Outer-surface protein A (OspA). In the current study, the recombinant OspA vaccine was chosen for its reported efficacy and safety profile and was recommended by the veterinary hospital as a 'CORE' vaccine for all dogs in 2009-2013. In each year analyzed, the risk of a dog testing seropositive for infection with Borrelia burgdorferi was greatly influenced by vaccine status; on average, over the 5-year study period just $1.01 \%$ (range of $0.39-1.32 \%$ ) of fully vaccinated dogs exhibited evidence of a newly acquired infection while $21.62 \%$ (range $16.8-32.08 \%$ ) of incompletely vaccinated dogs experienced a new infection [Table 3]. Using the modified frequency vaccination protocol wherein the scheduled first yearly booster was administered 6-months early, no adverse events were reported. Using Risk Ratio calculations, incompletely vaccinated dogs were between 14 and 52 times more likely to become seropositive for B. burgdorferi infection compared to their 'fully' vaccinated cohorts [Table 3]. In terms of a dog becoming newly positive for $B$. burgdorferi-specific antibodies (an indirect measure of infection), the Preventative Fraction when using the rOspA vaccine ranged from 93.29\% in 2009 and $98.08 \%$ in 2013 [Table 3]. One limitation of this analysis is that the effect of tick preventative use (acaracides) between fully and incompletely vaccinated dogs was not determined. One could hypothesize that those dogs most likely to complete their vaccine series and remain vaccine protocol-compliant might be dogs with owners who are more likely to purchase tick control. Even if both groups purchased tick preventative products, 
Table 3 The profile of a new-positive SNAP test result for Borrelia burgdorferi infection, including preventative fraction and risk ratio assessment among incompletely and completely vaccinated dogs

\begin{tabular}{|c|c|c|c|c|c|}
\hline & 2009 & 2010 & 2011 & 2012 & 2013 \\
\hline Percent $(+)$ of all tests run & $12.22 \%$ & $15.45 \%$ & $12.30 \%$ & $13.70 \%$ & $13.70 \%$ \\
\hline NEW (+) as $\%$ of all tests run & $5.90 \%$ & $7.86 \%$ & $5.60 \%$ & $7.01 \%$ & $6.10 \%$ \\
\hline \multicolumn{6}{|l|}{ Profile of a NEW (+) out of ALL new $(+)^{\prime}$ 's } \\
\hline Incomplete-vaccination & $86.59 \%(71 / 82)$ & $89.52 \%(94 / 105)$ & $85.92 \%(61 / 71)$ & $86.08 \%(68 / 79)$ & $95.45 \%(63 / 66)$ \\
\hline Full-vaccination & $13.41 \%(11 / 82)$ & $10.48 \%(11 / 105)$ & $14.08 \%(10 / 71)$ & $13.92 \%(11 / 79)$ & $4.55 \%(3 / 66)$ \\
\hline \multicolumn{6}{|l|}{ Infection rate (IR) based on vaccine status*: } \\
\hline ALL incomplete-vaccination & $16.86 \%(71 / 421)$ & $32.08 \%(94 / 293)$ & $18.32 \%(61 / 333)$ & $23.13 \%(68 / 294)$ & $20.32 \%(63 / 310)$ \\
\hline Complete-vaccination & $1.13 \%(11 / 970)$ & $1.04 \%(11 / 1053)$ & $1.07 \%(10 / 933)$ & $1.32 \%(11 / 833)$ & $0.39 \%(3 / 762)$ \\
\hline \multicolumn{6}{|l|}{ Negative infection rate based on vaccine status } \\
\hline ALL incomplete-vaccination & $83.14 \%(350 / 421)$ & $67.92 \%(199 / 293)$ & $81.68 \%(272 / 333)$ & $76.87 \%(226 / 294)$ & $79.68 \%(247 / 310)$ \\
\hline Complete-vaccination & $98.87 \%(959 / 970)$ & $98.96 \%(1042 / 1053)$ & $98.93 \%(923 / 933)$ & $98.68 \%(822 / 833)$ & $99.61 \%(759 / 762)$ \\
\hline Chi-square test statistic & 130.95 & 307.01 & 137.89 & 158.56 & 151.47 \\
\hline P-value & $<0.0001$ & $<0.0001$ & $<0.0001$ & $<0.0001$ & $<0.0001$ \\
\hline Preventative fraction (PF) against infection** & $93.29 \%$ & $96.76 \%$ & $94.16 \%$ & $94.29 \%$ & $98.08 \%$ \\
\hline Risk ratio $(\mathrm{RR})^{* * *}$ & 14.92 & 30.85 & 17.12 & 17.52 & 52.10 \\
\hline
\end{tabular}

*Infection Rate $=$ No. positive in vaccine status category/all dogs tested in that category. NOTE: All dogs other than those testing New (+) had (-) tests.

**PF $=[$ (Incidence of Event in Untreated - Incidence of Event in Treated)/Incidence of Event in Untreated $] \times 100$

${ }^{* * *}$ Risk Ratio $=\%$ Infected when incompletely vaccinated $/ \%$ infected when completely vaccinated.

it could be hypothesized that the fully compliant owners purchased, on average, more doses throughout the year. While additional analysis of the database may be useful to detect whether the variable of tick control played a role in Lyme-seropositive rates, the vast majority $(>80 \%)$ of all canine patients within the hospital database receive less than 9 months of tick protection each year, regardless of their vaccine status. Given that infected Ixodes scapularis can transmit Borrelia burgdorferi whenever they attach to a suitable host, regardless of season, the influence of tick control (or lack thereof) between well vaccinated and incompletely vaccinated may be minimal. Beyond tick control, perhaps an analysis of newly positive SNAP tests by owner zip codes would reveal that B. burgdorferi infection pressure is greater in certain areas leading to new infections prior to completion of the recommended vaccine protocol. Lastly, while the Preventative Fraction figures related to seropositivity and vaccination status are extremely compelling, they are not substitutes for data derived from prospective, randomized, placebo-controlled studies. To be clear, the data from the study described here were not randomly collected, so it is not a comprehensive representation of the whole population.

\section{Conclusions}

In this analysis, the practice owner's unique interest in data-mining the hospital's medical-record database to assess the rate of new $B$. burgdorferi-positive test results over a 5 year period is remarkable for its thoroughness and its findings. Furthermore, this analysis compliments and builds upon a previously published 21-month, 1,220 dog clinical observation study that showed that previously-negative, well vaccinated dogs rarely test positive for the presence of C6 antibodies specific for infection with B. burgdorferi [14]. In conclusion, for all the years from 2009 to 2013, the vaccination condition (e.g. 'fully' vaccinated versus not) and SNAP test results are not independent. We can therefore conclude that there is a strong, statistically significant relationship between compliance with this hospital's immunization protocol using the canine recombinant OspA vaccine and the markedly low incidence rate of new cases of $B$. burgdorferi-positive test results (SNAP) in dogs [21].

\section{Abbreviations}

SNAP: Brand name of IDEXX lyme antibody test kit; rOspA: Recombinant outer surface protein A; PF: Preventive fraction; RR: Risk ratio; Bb: Borrelia burgdorferi.

\section{Competing interests}

Financial: Dr. Andrew Eschner is a veterinarian employed by Merial; Dr. Kristen Mugnai is employed by Coastal Veterinary Care.

Non-Financial: Dr. Andrew Eschner has spoken at an IDEXX-sponsored Symposium and participated in an IDEXX-sponsored Webinar about vaccination and testing for canine Lyme borreliosis.

\section{Authors' contributions}

KM collected and analyzed 5-year patient data set. AKE further analyzed data and arranged the consultation services of the University of Georgia Statistical Consulting Center. AKE wrote the manuscript, prepared the tables and charts from the Excel Spreadsheets and e-mails supplied by KM. Both AKE and KM helped with results interpretation and were involved in editing, revising and approving the final version of the manuscript. 


\section{Authors' information}

KM is a 1996 graduate of Oregon State University College of Veterinary Medicine and has been the owner of Coastal Veterinary Care, a 3-doctor veterinary practice since 2005. She adopted the modified canine Lyme disease vaccine protocol recommendation by Straubinger and others in 2007 and asked the question in 2013 whether vaccinating for Lyme, in general, and adopting a slight variation on the typical vaccine protocol, in specific, was making a discernable difference to her canine patients. AKE is a 1990 graduate of Cornell University College of Veterinary Medicine and joined Merial as a technical services veterinarian in 1995 and resides in the Upstate New York area. He continues to strive to better understand the complexities of Lyme borreliosis, including its biology, diagnostics and preventative strategies.

\section{Acknowledgements}

The authors wish to thank G. Bion Richards who provided the contact point between AKE and KM and who forged the opportunity to meet and dialogue about the data. The authors also wish to acknowledge the University of Georgia Statistical Consulting Center including the statisticians Kim Love-Myers, Fangzhi Guo, and Yuanwen Wang for their time and expertise analyzing the data. AKE wishes to acknowledge and thank Prof. Dr. med. vet. Reinhard K. Straubinger, Ph.D. for his review of the manuscript. Funding for data collection and analysis of hospital software and client database is provided by KM. KM is the owner of Coastal Veterinary Care, Wiscasset, ME. Funding for the U. Georgia's statistical consulting services and manuscript publication is provided by Merial. AKE is employed by Merial, Duluth GA.

\section{Disclaimer}

This document is provided for scientific purposes only. Any references to a brand or trademark herein is for informational purposes only and is not intended for a commercial purpose or to dilute the rights of the respective owner(s) of the brand(s) or trademark(s).

SNAP ${ }^{\oplus}, 3 D X^{\circledast}, 4 D x^{\circledast}$ and $4 D X^{\circledast}$ Plus are registered trademarks of IDEXX Inc. in the United States and elsewhere.

RECOMBITEK ${ }^{\circledast}$ is a registered trademark of Merial in the United States and elsewhere.

\section{Author details}

${ }^{1}$ Merial Limited, 3239 Satellite Boulevard NW, Duluth, GA 30096, USA.

${ }^{2}$ Coastal Veterinary Care, 161 Gardiner Road, Wiscasset, ME 04578, USA.

Received: 13 November 2014 Accepted: 20 January 2015

Published online: 10 February 2015

\section{References}

1. Lissman BA, Bosler EM, Camay H, Ormiston BG, Benach JL. Spirochete associated arthritis (Lyme disease) in a dog. JAVMA. 1984;185:219-20.

2. Straubinger RK, Straubinger AF, Summers BA, Jacobson RH, Erb HN. Clinical manifestations, pathogenesis, and effect of antibiotic treatment on Lyme borreliosis in dogs. Wien Klin Wochenschr. 1998;110(24):874-81.

3. Stanek G, Fingerle V, Hunfeld KP, Jaulhac B, Kaiser R, Krause A, et al. Lyme borreliosis: clinical case definitions for diagnosis and management in Europe. Clin Microbiol Infect. 2011;17:69-79.

4. Piesman J, Schwan T. From ecology of borreliae and their arthropod vectors. In: Samuels RA, Radolf JD, editors. Borrelia: molecular biology, host interaction and pathogenesis. 1st ed. Norfolk UK: Caister Academic Press; 2010. p. 261-78.

5. Straubinger RK, Straubinger AF, Harter L, Jacobson RH, Chang YF, Summers BA, et al. Borrelia burgdorferi migrates into joint capsules and causes an up-regulation of interleukin-8 in synovial membranes of dogs experimentally infected with ticks. Infect Immun. 1997;65:1273-85.

6. Chu HJ, Chavez LG, Blumer BM, Sebring RW, Wasmoen TL, Acree WM. Immunogenicity and efficacy study of a commercial Borrelia burgdorferi bacterin. JAVMA. 1992;201:403-11.

7. LaFleur RL, Dant JC, Wasmoen TL, Callister SM, Jobe DA, Lovrich SD, et al. Bacterin that induces anti-OspA and anti-OspC borreliacidal antibodies provides a high level of protection against canine lyme disease. Clin Vacc Immun. 2009;16:253-9.
8. LaFleur RL, Callister SM, Dant JC, Jobe DA, Lovrich SD, Warner TF, et al. One-year duration of immunity induced by vaccination with a canine lyme disease bacterin. Clin Vacc Immun. 2010;17:870-4.

9. Wikle RE, Fretwell B, Jarecki M, Jarecki-Black JC. Canine lyme disease: one-year duration of immunity elicited with a canine OspA monovalent lyme vaccine. Intern J Appl Res Vet Med. 2006;4:23-8.

10. Conlon JR, Mather TN, Tanner P, Gallo G, Jacobson RH. Efficacy of a nonadjuvanted, outer surface protein $A$, recombinant vaccine in dogs after challenge by ticks naturally infected with Borrelia burgdorferi. Vet Ther. 2000;1:96-107.

11. Levy LA. Use of a C6 ELISA test to evaluate the efficacy of a whole-cell bacterin for the prevention of naturally transmitted canine Borrelia burgdorferi infection. Vet Thera. 2002;3:420-4.

12. Levy SA, Lissman BA, Ficke CM. Performance of a Borrelia burgdorferi bacterin in Borreliosis-endemic areas. JAVMA. 1993;202:1834-8.

13. Levy SA, Clark KK, Glickman LT. Infection rates in dogs vaccinated and not vaccinated with an OspA borrelia burgdorferi vaccine in a lyme diseaseendemic area of connecticut. Intern J Appl Res Vet Med. 2005;3:1-5.

14. Hebert D, Eschner A. Seroprevalence of Borrelia burgdorferi-specific C6 antibody in dogs before and after implementation of a recombinant, nonadjuvanted outer surface protein a vaccine in a Rhode island small animal clinic. Vet Thera. 2010;11:1-8.

15. Appel MJG, Jacobson RH. CVT update: canine lyme disease. In: Bonagura JD, editor. Kirk's current veterinary therapy XII. Philadelphia: WB Saunders; 1995. p. 303-9.

16. Moore GE, Guptill LF, Ward MP, Glickman NW, Faunt KK, Lewis HB, et al. Adverse events diagnosed within three days of vaccine administration in dogs. JAVMA. 2005;227:1102-8.

17. Topfer KH, Straubinger RK. Characterization of the humoral immune response in dogs after vaccination against the Lyme borreliosis agent - a study with five commercial vaccines using two different vaccination schedules. Vaccine. 2005;25:314-26.

18. Van HC, Lebacq E, Beran J, Parenti D. Alternative vaccination schedules $(0,1$ and 6 months versus 0, 1 and 12 months) for a recombinant OspA lyme disease vaccine. Clin Infect Dis. 1999;28:1260-4.

19. IDEXX company website [https://www.idexx.com/smallanimal/inhouse/ snap/4dx.html]

20. Measures of Association (Boston University School of Public Health). [http://sphweb.bumc.bu.edu/otlt/MPH-Modules/EP/EP713_Association/ EP713_Association_print.html]

21. University of Georgia Statistical Consulting Center. Comparison of lyme disease infection rates between fully and incompletely/non-vaccinated dogs. Athens: Fangzhi Guo, Yuanwen Wang, and Kim Love-Myers; 2014.

\section{Submit your next manuscript to BioMed Central and take full advantage of:}

- Convenient online submission

- Thorough peer review

- No space constraints or color figure charges

- Immediate publication on acceptance

- Inclusion in PubMed, CAS, Scopus and Google Scholar

- Research which is freely available for redistribution 